\title{
Heme oxygenase-1 mediates the protective effects of rapamycin in monocrotaline-induced pulmonary hypertension
}

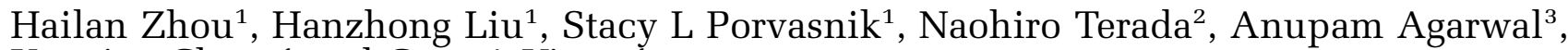 \\ Yanping Cheng ${ }^{1}$ and Gary A Visner ${ }^{1}$ \\ ${ }^{1}$ Department of Pediatrics, University of Florida, Gainesville, FL, USA; ${ }^{2}$ Department of Pathology, University \\ of Florida, Gainesville, FL, USA and ${ }^{3}$ Department of Medicine, University of Alabama-Birmingham, \\ Birmingham, AL, USA
}

\begin{abstract}
Rapamycin inhibits the development and progression of vascular disease. We previously showed that rapamycin induces the cytoprotective protein, heme oxygenase-1 (HO-1), and more importantly, chemically inhibiting HO-1 blocked the antiproliferative actions of rapamycin. In this study, we evaluated whether HO-1 is required for the vascular protective effects of rapamycin in vivo using a rat monocrotaline-induced pulmonary hypertension model. Rats were exposed to monocrotaline with or without rapamycin and $\mathrm{HO}$ activity was altered using the chemical inhibitor, tin protoporphyrin or the inducer, cobalt protoporphyrin. We also evaluated possible mechanisms of rapamycin-dependent induction of HO-1, and how HO-1 mediates growth factor-dependent antiproliferative actions of rapamycin. Proliferation and cell cycle progression were examined in smooth muscle cells derived from both wild-type and $\mathrm{HO}-1$ knockout $\left(\mathrm{HO}^{-1^{-l}}\right)$ mice in response to growth factors and rapamycin. Similar to our previous findings in vitro, rapamycin induced HO-1 in rat lung. Rapamycin also inhibited the development of monocrotaline-induced pulmonary hypertension, and this protective effect was blocked with the addition of tin protoporphyrin. In addition, treatment with cobalt protoporphyrin resulted in a substantial protection in this model of pulmonary hypertension. Rapamycin induction of HO-1 was dependent upon a transcriptional event; however, it was not mediated through an altered redox state or mammalian targets of rapamycin inhibition. Unlike wild-type cells, the growth of $\mathrm{HO}^{-1^{-1}}$ mouse aortic smooth muscle cells was not inhibited or cell cycle arrested in G1 in response to rapamycin. This study demonstrates that HO-1 is critical for the antiproliferative and vascular protective effects of rapamycin in vitro and in vivo in monocrotaline-induced pulmonary hypertension.
\end{abstract}

Laboratory Investigation (2006) 86, 62-71. doi:10.1038/labinvest.3700361; published online 31 October 2005

Keywords: animal model; aorta; heme oxygenase; monocrotaline; pulmonary hypertension; rapamycin

The fibroproliferative response to injury is a pathologic property of vascular diseases, such as pulmonary hypertension. ${ }^{1-3}$ Pathologic changes observed in pulmonary arterial hypertension (PAH) include medial hypertrophy, adventitial thickening, and abnormal growth of endothelial cells. A limitation of current therapies for PAH is that they are mostly aimed at vasodilatation, rather than against the fibroproliferative response. ${ }^{1,3}$ Rapamycin is an immunosuppressive agent with antiproliferative activity not only against lymphocytes but also against

Correspondence: Dr GA Visner, DO, Division of Pulmonology, Department of Pediatrics, University of Florida, Box 100296, 1600 SW Archer Road, Gainesville, FL 32610-0296, USA.

E-mail: visnega@peds.ufl.edu

Received 29 July 2005; revised 13 September 2005; accepted 14 September 2005; published online 31 October 2005 vascular cells. ${ }^{4-6}$ Since cellular proliferation is a hallmark of PAH, antiproliferative agents such as rapamycin may be an attractive therapeutic alternative. In fact, rapamycin reduces neointimal formation in a balloon injury model of vascular disease $^{7,8}$ and is used in stents to prevent vascular restenosis. ${ }^{9}$ Rapamycin has been shown to attenuate pulmonary hypertension and neointimal formation in a rat model of PAH. ${ }^{10}$ The mechanisms underlying these protective effects are unknown, but they are unlikely to be the result of rapamycin's immunosuppressive properties, since other immunosuppressive agents have no protective effects in $\mathrm{PAH},{ }^{11}$ and do not inhibit growth factor-dependent proliferation of vascular cells. ${ }^{12}$

Rapamycin binds to FK-binding protein 12, and this complex binds to mammalian targets of rapamycin (mTOR) leading to inhibition of both DNA 
and protein synthesis and cell cycle arrest. ${ }^{13}$ The antiproliferative effects of rapamycin on mesenchymal cells led to its use in fibroproliferative vascular disorders. However, its full spectrum of actions and the exact mechanisms underlying its vascular protective effects have not been elucidated. We reported that rapamycin induces the cytoprotective protein heme oxygenase-1 (HO-1) and inhibits growth factor-dependent proliferation of human pulmonary artery smooth muscle cells (HPASMC). More importantly, we showed that rapamycin required HO-1 for its antiproliferative effects, since chemical inhibition of HO activity with tin protoporphyrin resulted in a loss of its effects on smooth muscle cell (SMC) proliferation. ${ }^{14}$

$\mathrm{HO}$ is the rate-limiting enzyme in the degradation of heme to biliverdin, and subsequently to bilirubin. ${ }^{15}$ There are two major isoenzymes of $\mathrm{HO}$, with $\mathrm{HO}-1$ being the inducible isoform and HO-2 the constitutive isoform. HO-1 induction is believed to be a cytoprotective response, and has been shown to have vasodilatory, anti-inflammatory, and antiapoptotic properties. ${ }^{16,17}$ An increase in HO-1 prevented hypoxia-induced pulmonary hypertension and inflammation. ${ }^{18}$ Concomitantly, a lack of HO-1 expression (HO-1 knockout mice) is associated with organized mural thrombi and subsequent pulmonary hypertension in response to hypoxia. ${ }^{19}$

In this study, we evaluated whether rapamycin's vascular protective effects in vivo were possibly mediated through HO-1 using a rat monocrotaline model of PAH. In addition, we further evaluated the role of HO-1 in mediating the antiproliferative effects of rapamycin in aortic SMC derived from HO-1-deficient $\left(\mathrm{HO}^{-1-}{ }^{-1}\right)$ and wild-type mice.

\section{Materials and methods}

\section{Material Supplies}

Monocrotaline, platelet-derived growth factor (PDGF), N-acetylcysteine (NAC), hemin, actinomycin D, and cycloheximide were purchased from Sigma, St Louis, MO, USA. Rapamycin was provided by Weyth Research, Monmouth Junction, NJ, USA. Tin (IV) and cobalt (III) protoporphyrin IX chloride (SnPP and CoPP) were purchased from Frontier Scientific, Logan, UT, USA.

\section{Animal Model}

Male Sprague-Dawley rats were purchased from Harlan, Indianapolis, IN, USA. The investigation conforms with the Guide for the Care and Use of Laboratory Animals published by the US National Institutes of Health. Control groups received subcutaneous injection of vehicle and olive oil by gavage. Monocrotaline, $100 \mathrm{mg} / \mathrm{kg}$, was given by subcutaneous injection, and rapamycin, $2 \mathrm{mg} / \mathrm{kg}$, was given daily by gavage starting 1 day prior to monocrotaline injection. Preliminary studies in our laboratory demonstrated that $2 \mathrm{mg} / \mathrm{kg}$ of rapamycin resulted in a $24 \mathrm{~h}$ trough level of $\sim 5 \mathrm{ng} / \mathrm{ml}$. SnPP and CoPP, $20 \mu \mathrm{mol} / \mathrm{kg}$, were given by subcutaneous injection on days 5 and 15 . The following are the number of animals per experimental group: control $=$ six; rapamycin $=$ four; monocrotaline $=$ six; monocrotaline + rapamycin $=$ six; monocrotaline + rapamycin $+\mathrm{SnPP}=$ six; monocrotaline $+\mathrm{SnPP}=$ six; $\mathrm{SnPP}=$ four; $\mathrm{CoPP}=$ four; monocrotaline $+\mathrm{CoPP}=$ six.

After 28 days, hemodynamic measurements were made and animals were killed. Rats were anesthetized with isoflurane/oxygen, intubated orotracheally, and ventilated with room air/isoflurane. The heart was exposed through a midline chest incision, and a Millar catheter inserted through the right ventricle into the pulmonary artery. Pulmonary artery pressure $(\mathrm{PaP})$ was monitored and data were collected using PowerLab system and software (ADInstruments, Colorado Springs, CO, USA). The lungs were then flushed until clear with ice-cold saline at $20 \mathrm{~cm} \mathrm{H}_{2} \mathrm{O}$. The right lung and heart was removed and the right ventricle (RV) separated from the left ventricle (LV) and septum (S), and weighed. The right lung was frozen in liquid nitrogen and stored at $-80^{\circ} \mathrm{C}$. The left lung was filled with $10 \%$ buffered formalin, removed, fixed overnight, and paraffin embedded. Sections $(5 \mu \mathrm{m})$ were cut at two levels in the paraffin block and stained with a standard hematoxylin-eosin stain, and additional staining was performed using Verhoeff-van Gieson stains to highlight elastic lamina. Neointimal formation was determined by computerized morphometry ${ }^{20}$ and is represented as lumen size to total vessel size, calculated by area within the internal elastic lamina/area within external elastic lamina $\times 100$ using image analysis software (Zeiss Axioplan 2 with SPOT digital camera and image analysis, Diagnostic Instruments Inc., Sterling Heights, MI, USA).

\section{Cell Culture}

HPASMC (Clonetics, Walkersville, MD, USA) were grown in SmGM media (SmBM-basal media plus growth factors, Clonetics) with $10 \%$ fetal bovine serum (FBS). Human alveolar rhabdomyosar coma (Rh30) cells expressing wild-type mTOR or rapamycin-resistant mTOR were obtained from PJ Houghton (St Jude, Memphis, TN, USA) ${ }^{21}$ and maintained in DMEM with $10 \%$ FBS. Mouse aortic SMC (mSMC) were isolated from wild-type or $\mathrm{HO}-1^{-1-}$ mice by collagenase and elastase treatment of isolated aortas. Cells were resuspended in SmGM media containing $10 \% \mathrm{FBS}$ in room air $/ 5 \% \mathrm{CO}_{2}$ at $37^{\circ} \mathrm{C}$. SMC cells were verified by staining for $\alpha$ smooth muscle actin $(0.5 \mathrm{mg} / \mathrm{ml}$ mouse monoclonal $\alpha$-actin, FITC, Abcam, Cambridge, UK) and identified under a fluorescence microscope. $\mathrm{HO}-1^{-1-}$ mice (C57BL/6 background) were originally from Susumu 
Tonegawa's laboratory (Massachusetts Institute of Technology, Cambridge, MA, USA). ${ }^{22}$ Owing to a low yield of homozygous mice, the original breeding colony was backcrossed with FVB to derive a HO-1 ${ }^{-/-}$colony with a predominant FVB background resulting in an increased yield of homozygous mice. ${ }^{23}$

\section{Protein Isolation and Western Analysis}

In all, $10^{6}$ cells $/ \mathrm{ml}$ were washed and lysed with Triton lysis buffer. Frozen lung tissue was homogenized in $20 \mathrm{mM} \mathrm{KPO}$ buffer ( $\mathrm{pH} 7.4), 0.1 \mathrm{mM}$ EDTA, $135 \mathrm{mM} \mathrm{KCl}$, and the homogenate was sonicated, centrifuged, and resuspended in $20 \mathrm{mM}$ $\mathrm{KPO}_{4}$ buffer (pH 7.4), $10 \mathrm{mM}$ EDTA, $1 \mathrm{mM} \mathrm{KCl}$. Total protein $(30 \mu \mathrm{g})$ was separated using a 10\% SDSpolyacrylamide gel and transferred to a polyvinylidene difluoride membrane (Immobilon ${ }^{\mathrm{TM}}$-P, Milipore, Bedford, MA, USA). The membranes were blocked with $5 \%$ nonfat dry milk in $10 \mathrm{mM}$ Tris $\mathrm{pH}$ 7.5, $100 \mathrm{mM} \mathrm{NaCl}$, and $0.05 \%$ Tween 20 and incubated for $1.5 \mathrm{~h}$ with mouse anti-P21 antibody (Santa Cruz, California, CA, USA), rabbit anti-P27 antibody, mouse anti-Cyclin D1 (Cell Signaling Technology, Beverly, MA, USA), or rabbit anti-HO1 (StressGen, Victoria, BC, Canada). The proteins were visualized using a secondary antibody, peroxidase-conjugated goat anti-rabbit and anti-mouse IgG antibody, and chemiluminescence according to the manufacturer's recommendations (Pierce, Rockford, IL, USA).

\section{RNA Isolation and Northern Analysis}

Total RNA was isolated and purified using RNeasy mini kits (Qiagen, Valencia, CA, USA). The RNA was fractionated by size on a $1 \%$ agarose, $6 \%$ formaldehyde gel buffered by 3-( $N$-morpholino) propanesulfonic acid, electrotransferred to a nylon membrane, and crosslinked with ultraviolet light. The RNA was hybridized to ${ }^{32} \mathrm{P}$-labeled cDNAs, HO1, or glyceraldehyde 3-phosphate dehydrogenase (GAPDH) as an internal control. The membranes were subjected to autoradiography using an intensifying screen at $-80^{\circ} \mathrm{C}$.

\section{Plasmid Constructs and Transient Transfection Analysis}

HO-1 promoter activity was evaluated using -11.6 and $-4.5 \mathrm{~kb}$ human $\mathrm{HO}-1$ promoter constructs (pHOGL3/-11.6 or -4.5 ) as previously described. ${ }^{24}$ A batch transfection protocol was performed in HPASMC using Lipofectamine (Invitrogen, Carlsbad, CA, USA) as previously described. ${ }^{25}$ The day after transfection, cells were split into smaller plates and then allowed to recover for $24 \mathrm{~h}$ before stimulation in $1 \%$ FBS in complete media with or without rapamycin or hemin for $16 \mathrm{~h}$. Cell lysates were collected and luciferase activity was measured according to the manufacturer's instructions (Promega, Madison, WI, USA).

\section{Analysis of Intracellular Reactive Oxygen Species}

HPASMC in media with $1 \%$ FBS were treated with a general oxidant scavenger, $10 \mathrm{mM}$ NAC $1 \mathrm{~h}$ prior to treatment with or without rapamycin $(10 \mu \mathrm{M})$ for $4 \mathrm{~h}$, and evaluated using 2'-7'-dichlorofluorescein-diacetate (DCF-DA) (Molecular Probes, Eugene, OR, USA) at a final concentration of $2 \mu \mathrm{M}$ and incubated for $45 \mathrm{~min}$ at $37^{\circ} \mathrm{C}$. Following $30 \mathrm{~min}$, cells were washed and harvested, and fluorescence was determined by flow cytometry (FACS-SCAN, Becton-Dickinson, Franklin Lakes, NJ, USA).

\section{Cell Proliferation Assay}

Two $\times 10^{3}$ wild-type or $\mathrm{HO}-1^{-/-}$mSMC were split into 96-well plates with SmGM plus 10\% FBS for $24 \mathrm{~h}$. The cells were placed in SmGM containing 1\% FBS for another $24 \mathrm{~h}$, then treated with PDGF (10 ng/ $\mathrm{ml})$, rapamycin $(10 \mu \mathrm{M})$, or SmGM with $10 \%$ FBS for $48 \mathrm{~h}$. Proliferation was evaluated using the tetrazolium-based assay (XTT) and 5-bromo-2-deoxyuridine (Brdu) cell proliferation kits (Roche, Indianapolis, IN, USA) according to the manufacturer's instructions.

\section{Cell Cycle Analysis}

Five $\times 10^{5}$ wild-type or $\mathrm{HO}-1^{-/-} \mathrm{mSMC}$ were plated in $100 \mathrm{~mm}$ plates and incubated in SmGM 10\% FBS for $24 \mathrm{~h}$, then starved in SmGM 1\% FBS for another $24 \mathrm{~h}$. Cell cycle phase was evaluated by FACS analysis with propidium iodide (PI) following $48 \mathrm{~h}$ of treatment; SmGM in 1\% FBS with or without PDGF (10 ng/ml), rapamycin $(10 \mu \mathrm{M})$, or SmGM in $10 \%$ FBS.

\section{Statistics}

Data are expressed as mean \pm s.e.m., and statistical analyses performed using the Prism statistical program (GraphPad, San Diego, CA, USA). Oneway ANOVA with the Tukey-Kramer multiple comparisons test was used to evaluate differences between groups. Linear correlations were evaluated using Pearson $r$. A $P$-value $<0.05$ was considered significant.

\section{Results}

We previously reported that the chemical inhibitor of HO-1, SnPP, inhibited the antiproliferative actions of rapamycin in HPASMC, ${ }^{14}$ suggesting that the vascular protective effects of rapamycin may be 
dependent upon HO-1. To verify whether HO-1 plays a critical role in rapamycin's protective effects in vascular disease, we used a rat monocrotalineinduced PAH model. Similar to our in vitro studies,${ }^{14}$ rapamycin induces lung HO-1 expression. Rapamycin increases HO-1 protein levels approximately three-fold following 5 days of exposure (Figure 1). Interestingly, cotreatment of the rats with SnPP resulted in a loss of HO-1 induction in rapamycin + monocrotaline-treated animals. Nishimura et $a l^{10}$ previously demonstrated that rapamycin inhibited monocrotaline-induced PAH. We also found that rapamycin attenuated the increase in $\mathrm{PaP}$ and neointimal formation in response to monocrotaline (Figure 2). In this study, we examined neointimal formation based on lumen size (luminal area/ total vessel area). We found a strong negative correlation of lumen size with $\mathrm{PaP}, P<0.0001$ (Figure 2c). More importantly, we found that inhibiting HO-1 using SnPP resulted in a loss of the protective effects of rapamycin against monocrotaline-induced disease, indicating a role of HO- 1 in the cytoprotective actions of rapamycin in vivo. In addition, inducing HO-1 with CoPP resulted in a dramatic inhibition of monocrotalineinduced $\mathrm{PAH}$ with both $\mathrm{PaP}$ and lumen size being similar to normal vessels, thereby demonstrating the protective effects of $\mathrm{HO}-1$ in pulmonary vascular disease.

A correlate to increased $\mathrm{PaP}$ is right ventricular hypertrophy (RVH), which is represented based on
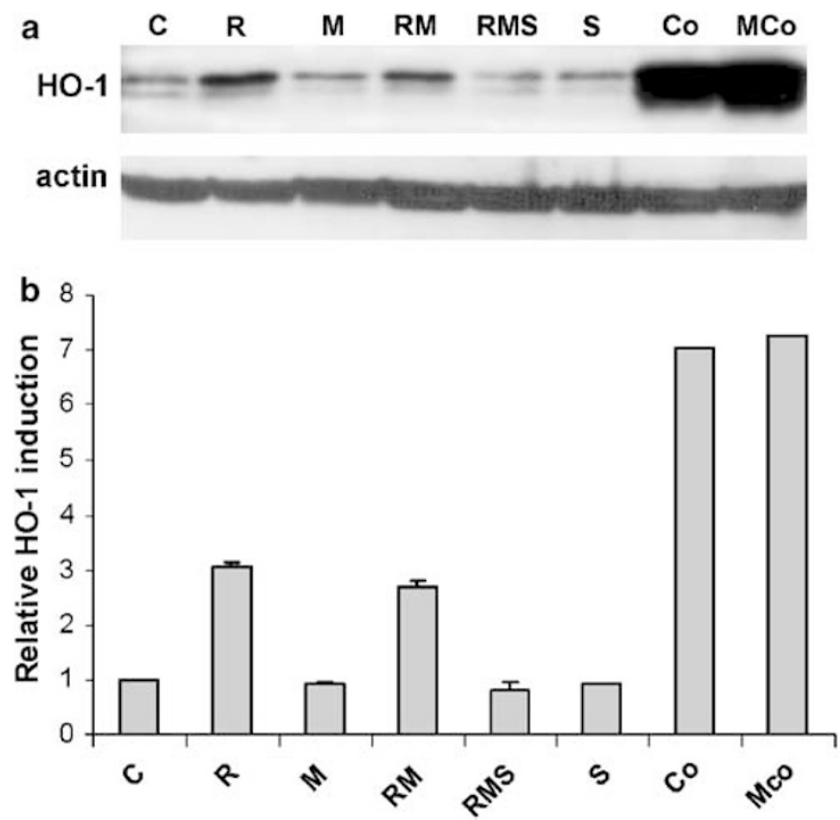

Figure 1 (a) Representative Western analysis of HO-1 protein levels at 5 days from untreated rats (control, C), rapamycin (R), monocrotaline (M), SnPP (S), and/or CoPP (Co). (b) Graph illustrating relative fold increase of $\mathrm{HO}-1$ protein in rat lung after 5 days of treatments. $N=3$ for C, R, M, RM, and $N=2$ for RMS, S, Co, and MCo.
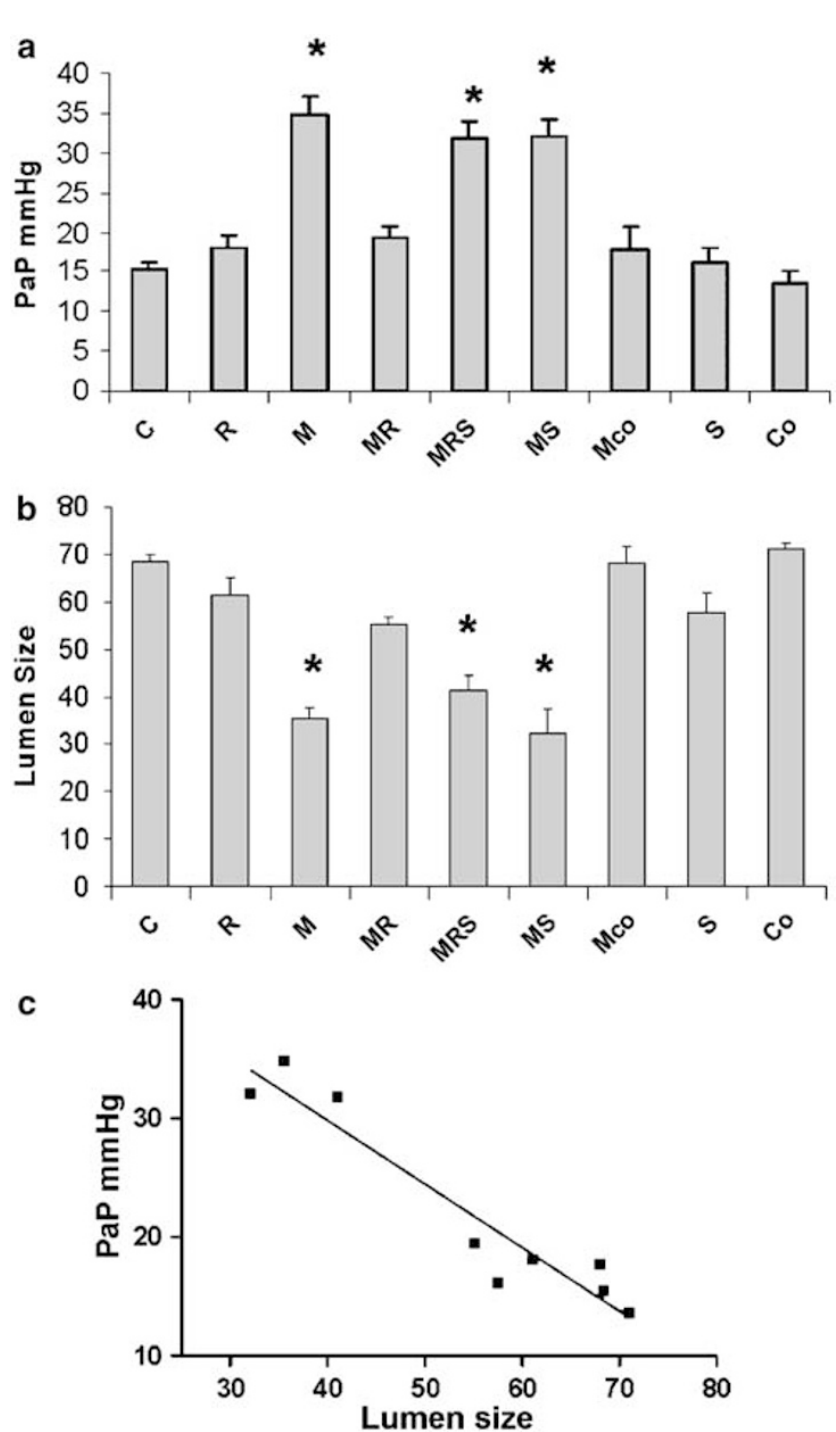

Figure 2 Rat monocrotaline model of pulmonary hypertension evaluated 28 days following monocrotaline treatment. (a) Graph of $\mathrm{PaP}$ for the following experimental conditions; vehicle-olive oil (C), rapamycin (R), monocrotaline (M), monocrotaline + rapamycin (MR), monocrotaline + rapamycin + SnPP (MRS), monocrotaline + SnPP (MS), moncrotaline + CoPP (Mco), SnPP (S), or CoPP (Co). $N=4-6$ animals for each condition. There was a significant difference for control, rapamycin, monocrotaline + rapmaycin, monocrotaline + CoPP, SnPP, and CoPP as compared to monocrotaline, monocrotaline + rapamycin, and monocrotaline + rapamycin $+\operatorname{SnPP}\left(P<0.01^{*}\right)$. (b) Graph of neointimal formation, percentage of lumen size compared to vessel size. Similar to PaP, there was a significant difference $\left(P<0.01^{*}\right)$ for control, rapamycin, monocrotaline + rapamycin, monocrotaline + CoPP, SnPP, and CoPP as compared to monocrotaline, monocrotaline + SnPP, and monocrotaline + rapamycin + SnPP. (c) The correlation between lumen size and $\mathrm{PaP}$ with Pearson $r-0.9541$ and $P<0.0001$. (d) Verhoff-Giesen stain (original magnification $\times 200$ ) of rat lung illustrating a representative pulmonary artery with monocrotaline treatment. (e) Verhoff-Giesen stain $(\times 200)$, monocrotaline + rapamycin treatment. (f) Verhoff-Giesen stain $(\times 200)$, monocrotaline + rapamycin + SnPP treatment. (g) Verhoff-Giesen stain $(\times 200)$, monocrotaline + CoPP.

RV weight to $L V$ plus S weight (Figures 3a and b). As expected, there was a highly significant correlation of $\mathrm{PaP}$ to $\mathrm{RV} / \mathrm{LV}+\mathrm{S}$. Both rapamycin and CoPP 

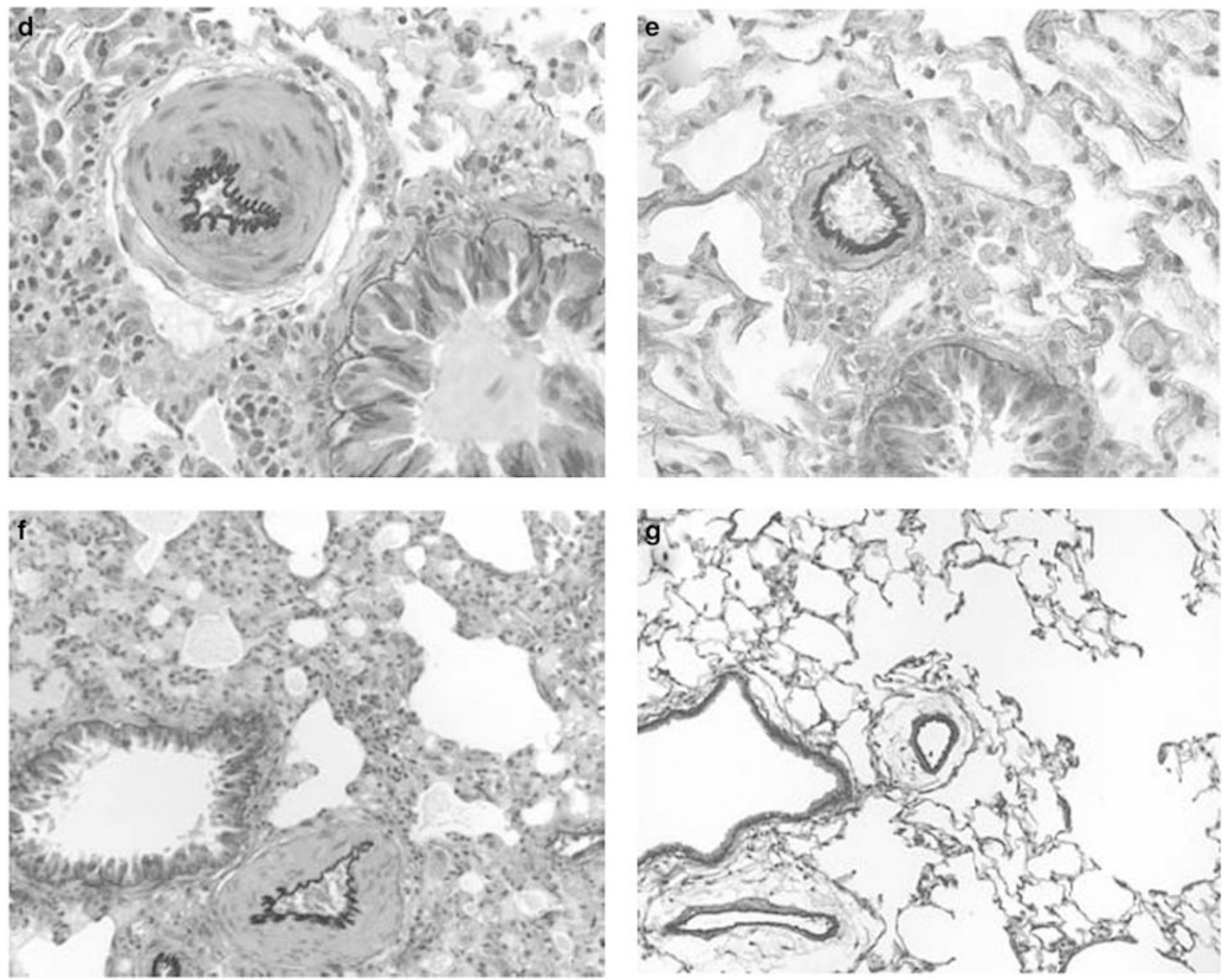

Figure 2 Continued.

attenuated monocrotaline-induced RVH. Unlike PaP and neointimal formation, rapamycin-dependent inhibition of monocrotaline-induced $\mathrm{RVH}$ was not blocked with SnPP. This does not appear to be related to the addition of SnPP since monocrotaline $+\mathrm{SnPP}$ had elevated $\mathrm{PaP}$ and $\mathrm{RV} / \mathrm{LV}+\mathrm{S}$.

To better evaluate the role of HO-1 in the antiproliferative effects of rapamycin, we isolated mSMC from wild-type and $\mathrm{HO}-1^{-/-}$mice (Figure 4a). Similar to human SMC, mSMC lacking HO-1 are not responsive to the antiproliferative actions of rapamycin. Figures $4 \mathrm{~b}$ and $\mathrm{c}$ show wild-type and HO- $1^{-1-}$ mSMC exposed to PDGF and proliferation evaluated with and without rapamycin. PDGF induced proliferation of both wild-type and $\mathrm{HO}-1^{-1-}$ mSMC. As expected, rapamycin inhibited PDGFdependent growth of wild-type mSMC; however, rapamycin had little effect on PDGF-stimulated growth of $\mathrm{HO}-1^{-1-} \mathrm{mSMC}$.

The antiproliferative actions of rapamycin are a result of blocking cell cycle progression in $\mathrm{G} 1^{13}$ and this was observed in wild-type mSMC (Figure 4d). However, rapamycin failed to arrest cell cycle progression in $\mathrm{HO}-1^{-/-} \mathrm{mSMC}$. Rapamycin inhibits cell cycle progression via upregulation of the cyclindependent kinase inhibitor, p27 ${ }^{\text {kip } 1} \cdot{ }^{26}$ We observed similar expression of p27 and the cell cycle proteins p21 and cyclin D1 in both wild-type and $\mathrm{HO}-1^{-/-}$ mSMC, indicating a p27-independent process (Figure 4e).

HO-1 induction by a variety of stimuli is dependent upon a transcriptional event. ${ }^{27}$ We found this to be also true in response to rapamycin (Figure 5a). Rapamycin-dependent induction of HO-1 mRNA is inhibited using actinomycin D, RNA synthesis inhibitor; however, there was little difference in HO-1 mRNA in response to cycloheximide, a protein synthesis inhibitor. We also observed an increase in HO-1 promoter activity in response to rapamycin similar to the response observed with hemin although to a lesser degree (Figure 5b).

Rapamycin inhibits mTOR, and we previously showed that wortmannin, an inhibitor of the upstream kinase PI-3K, also induced HO-1 expression. ${ }^{14}$ However, we found that LY294002, a more selective inhibitor of PI-3K had no effect on HO-1 expression (data not shown). To determine if HO-1 induction by rapamycin was through mTOR inhibi- 


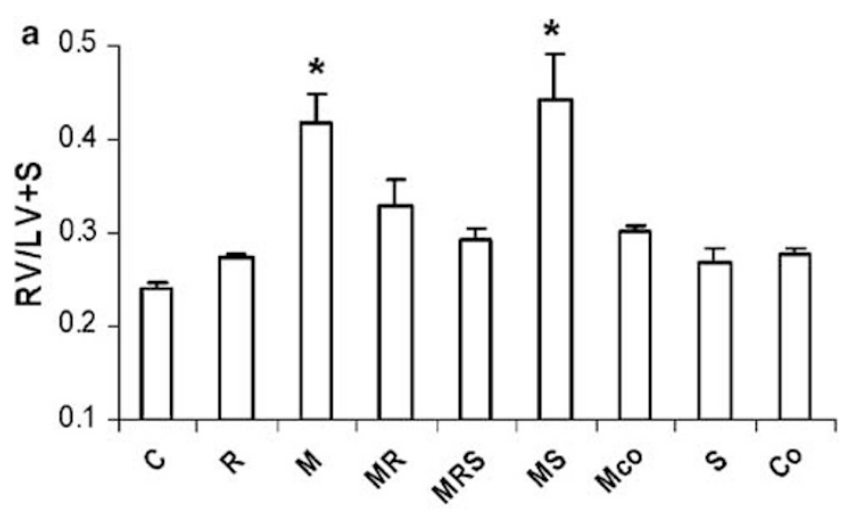

b

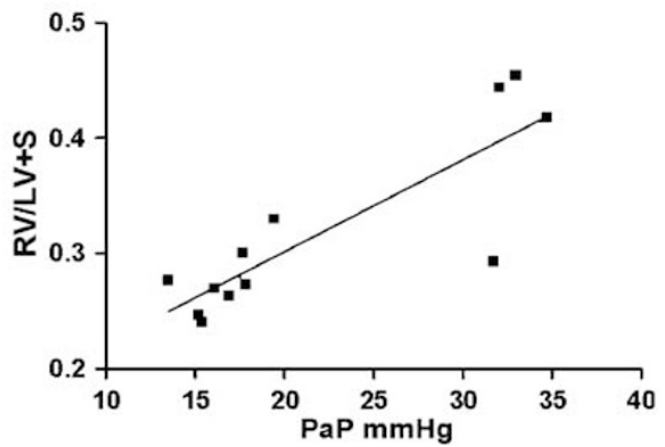

Figure 3 Cardiac remodeling. (a) Graph illustrating the assessment of the right heart $(\mathrm{RV} / \mathrm{LV}+\mathrm{S})$ under the various treatments. Again monocrotaline alone and monocrotaline $+\mathrm{SnPP}$ are significantly different $\left(P<0.05^{*}\right)$ from the other groups; however, unlike $\mathrm{PaP}$ and lumen size measurements, monocrotaline + rapamycin $+\mathrm{SnPP}$ treated group does not show a difference. (b) Graph showing the relationship between $\mathrm{RV} / \mathrm{LV}+\mathrm{S}$ to $\mathrm{PaP}$ showing a significant correlation, $P<0.0005$.

Figure 4 Wild-type (WT) and HO-1 ${ }^{-/-}$(KO) aortic mSMC, data are shown as mean \pm s.e.m. for three or more independent experiments for each condition. (a) Western analysis of $\mathrm{HO}-1^{-1-}$ or WT mSMC showing a lack of HO-1 expression in the $\mathrm{HO}^{-1}{ }^{-1}$ SMC with actin shown as an internal control. (b) Cell proliferation using XTT assay for the following treatment groups: maintained in $1 \%$ FBS (1\%); $1 \%$ FBS plus $10 \mu \mathrm{M}$ rapamycin (Rap); $1 \%$ FBS plus $10 \mathrm{ng} / \mathrm{ml}$ PDGF; $1 \%$ FBS, rapamycin and PDGF; and 10\% FBS. $P<0.001(*)$ for WT mSMC $1 \%$, Rap, or Rap/PDGF as compared to PDGF or $10 \%$ FBS, $P<0.001$ (\#) for $\mathrm{HO}-1^{-1-}$ mSMC $1 \%$ or Rap to PDGF or $10 \%$ as compared to PDGF or $10 \%$, while PDGF to Rap/PDGF was not significantly different $(P>0.05)$. (c) Cell proliferation based on DNA synthesis (Brdu assay) with experimental conditions as described above. $P<0.05\left({ }^{*}\right)$ for WT mSMC $1 \%$, Rap, or Rap/PDGF as compared to PDGF or $10 \%$, $P<0.05$ (\#) for HO-1 ${ }^{-1-}$ mSMC $1 \%$ or Rap to PDGF or $10 \%$, there was no significant difference for $\mathrm{HO}-1^{-1-}$ cells treated with PDGF as compared to Rap/PDGF. (d) Graph showing cell cycle phase based on FACS analysis with PI staining. WT and HO-1 $1^{-1-}$ mSMC demonstrated an increased number of cells in G0-G1 phase in 1\% FBS with a decrease following the addition of PDGF. The addition of rapamycin (Rap) resulted in an increase G0-G1 in WT mSMC $(P<0.01$ PDGF compared to Rap/PDGF); however, there was no significant change in HO-1 ${ }^{-/-}$SMC. (e) Representative Western analyses of p27, p21, cyclin D1, and actin from WT and HO-1 ${ }^{-1-}$ mSMC exposed to SmGM (SmBM plus growth factors and $10 \%$ FBS), growth factor withdrawal (only SmBM, GFW), and/or $10 \mu \mathrm{M}$ rapamycin. Lanes 1-7 are from $\mathrm{HO}^{-1-} \mathrm{mSMC}$ and lanes $8-14$ are wild-type (WT) mSMC: lanes 1 and 8-GFW $0 \mathrm{~h}$; lanes 2 and 9GFW $24 \mathrm{~h}$; lanes 3 and 10-GFW $48 \mathrm{~h}$; lanes 4 and 11-GFW for $24 \mathrm{~h}$ followed by SmGM $10 \%$ FBS for $24 \mathrm{~h}$; lanes 5 and $12-$ rapamycin; lanes 6 and 13-rapamycin for $24 \mathrm{~h}$ followed by SmGM $10 \%$ FBS for $24 \mathrm{~h}$; lanes 7 and $14-$ SmGM $10 \%$ FBS throughout the time period.

tion, we examined HO-1 expression in Rh30 cells expressing either wild-type or a rapamycin-resistant mutant of mTOR. HO-1 expression was similar in

a

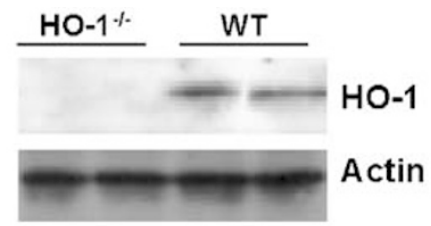

b

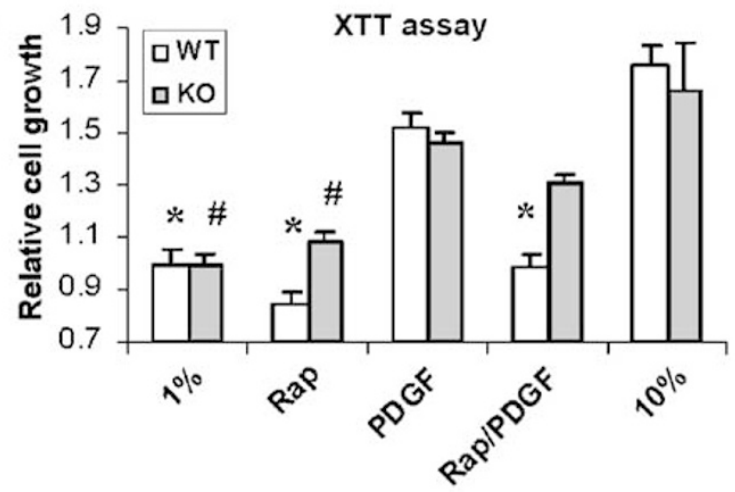

C

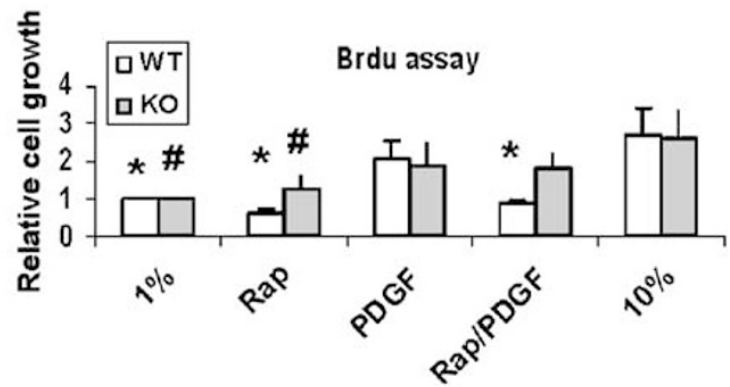

d

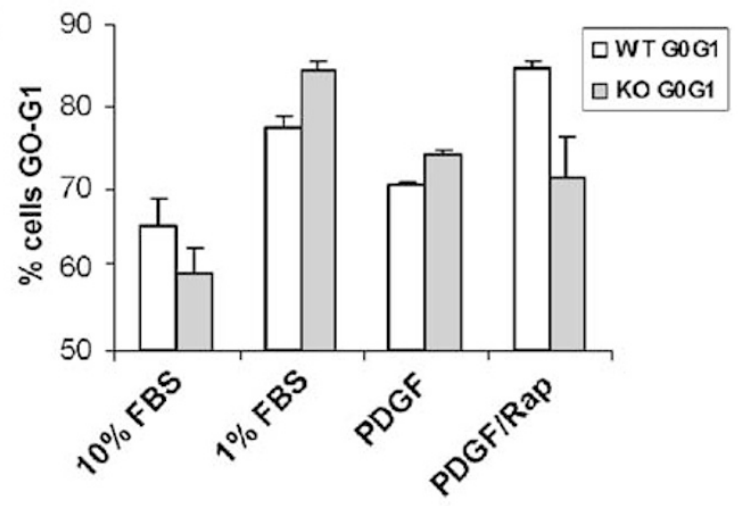

e

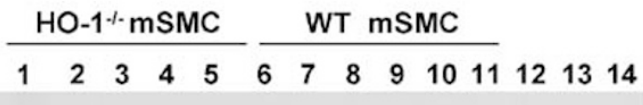

p27 kip1

p21Waf1/Cip1

Cyclin D1

Actin

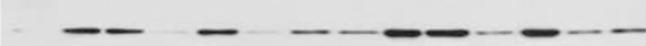

\section{.}

$$
\text { - }
$$




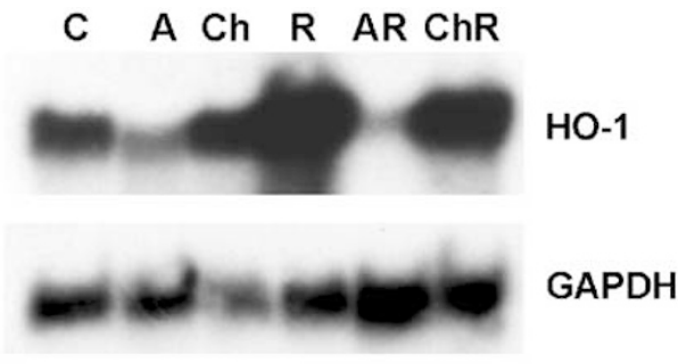

b
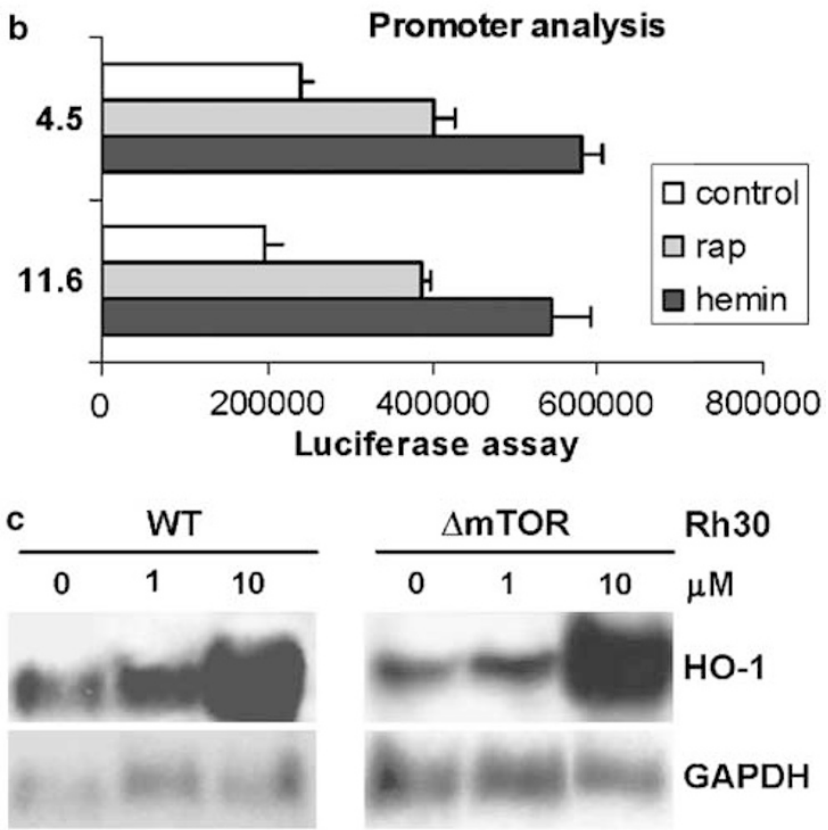

Figure 5 (a) Representative Northern analysis of HPASMC illustrating HO-1 and GAPDH mRNA levels in untreated (C) cells and those exposed to $5 \mu \mathrm{M}$ actinomycin D (A), $10 \mu \mathrm{M}$ cycloheximide (Ch), and/or $10 \mu \mathrm{M}$ rapamycin (R) for $4 \mathrm{~h}$. (b). Graph illustrating the mean \pm s.e.m. ( $N=3$ independent experiments) of HO-1 promoter activity using -11.6 and $-4.5 \mathrm{~kb}$ human HO-1 promoter constructs (pHOGL3/-11.6 or -4.5 ) in untreated cells (control) or exposed to $10 \mu \mathrm{M}$ rapamycin (rap), or $5 \mu \mathrm{M}$ hemintreated HPASMC. (c) Representative Northern analyses of wildtype (WT) and mTOR mutant ( $\Delta \mathrm{mTOR}) \mathrm{Rh} 30$ cells exposed to 0,1 , or $10 \mu \mathrm{M}$ rapamycin (Rap) showing an increase of HO-1 mRNA levels in both cell types. GAPDH is shown as an internal control for RNA loading.

both wild-type and mTOR-deficient Rh30 cells in response to rapamycin, thereby indicating an mTOR-independent mechanism of rapamycin (Figure 5c).

HO-1 is induced by oxidants and is a marker of oxidative stress ${ }^{28}$ leading to the possibility that rapamycin-mediated induction of HO-1 results from an altered redox state. We found that rapamycin increases cellular oxidation based on DCF analysis and this was completely inhibited with the antioxidant NAC (Figure 6a). However, pretreating cells with NAC had relatively little attenuation of HO-1 by rapamycin, indicating that its effect on HO-1 is at most partially mediated by an altered redox state (Figure $6 \mathrm{~b}$ and $\mathrm{c}$ ).
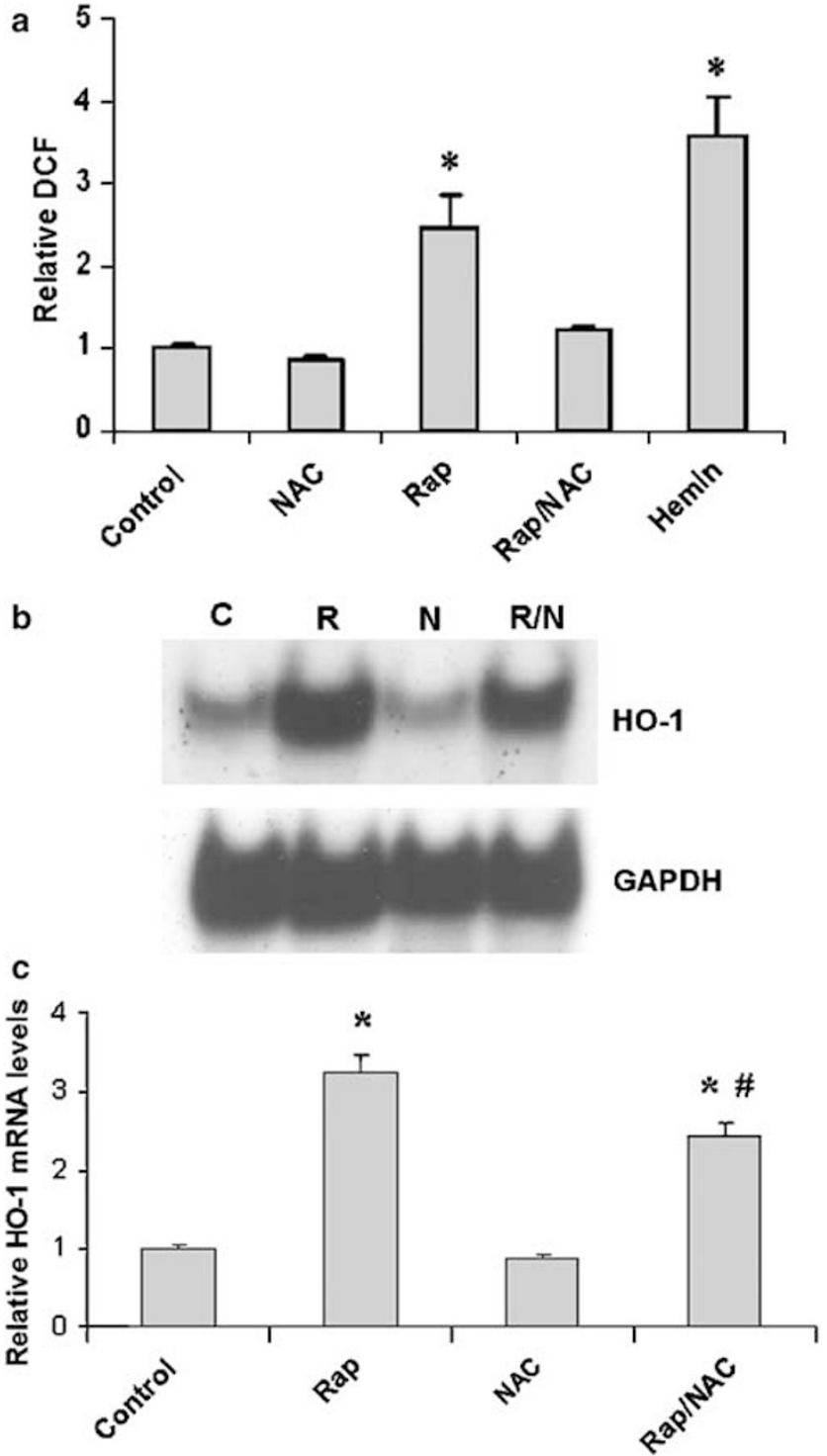

Figure 6 (a) Graph showing the relative change of HPASMC exposed to rapamycin (Rap) with or without NAC, untreated (control), or $5 \mu \mathrm{M}$ hemin (positive control) and evaluated for oxidant stress based on DCF analysis. Shown as mean \pm s.e.m. for $n=5$ except for hemin in which $n=3, P<0.05(*)$ for control, NAC, and Rap/NAC as compared to Rap and hemin. (b) Representative Northern analysis of HPASMC exposed to rapamycin (R) for $4 \mathrm{~h}$ with or without NAC (N) pretreatment illustrating HO-1 mRNA and GAPDH mRNA levels. C represents untreated (basal) HO-1 mRNA levels. (c) Graph illustrating relative fold increase in response to rapamycin (Rap) and/or NAC with $N=3$ per treatment group. Shown as mean \pm s.e.m. for $n=3$ for each experimental condition, $P<0.001\left({ }^{*}\right)$ for control or NAC to Rap or Rap/NAC and $P<0.05$ (\#) for Rap to Rap/NAC.

\section{Discussion}

Vascular remodeling is a hallmark in the progression of PAH. ${ }^{1,2}$ Eventually, these changes result in irreversible increases in PVR and right heart failure. Therefore, developing therapies that inhibit or reverse vascular remodeling is critical for the long-term management of PAH. Rapamycin is an 
immunosuppressive agent with potent vascular antiproliferative activities and is effective in preventing neointimal formation..$^{7-10}$ In fact, the implantation of rapamycin-coated stents has been shown to be safe and effective in preventing neointimal formation following stent placement in coronary artery disease. ${ }^{7-9}$ Recently, the potential benefit of rapamycin in PAH was illustrated in a rat monocrotaline model. ${ }^{10}$ However, the mechanisms underlying these effects remain unknown. Our laboratory recently found that rapamycin is an inducer of HO-1, which by itself has been shown to be protective against vascular remodeling. ${ }^{18,29}$ Furthermore, our previous data suggest that HO-1 is required for the antiproliferative effects of rapamycin.

In this study, we verified the findings that rapamycin not only inhibits systemic vascular disorders, but also protects against pulmonary vascular disease blocking both an increase in $\mathrm{PaP}$ and neointimal formation. We found that rapamycin exposure increased lung HO-1 expression prior to the onset of significant injury from monocrotaline. The induction of HO-1 in rapamycin- and monocrotaline-treated animals was inhibited by SnPP, so that it not only acted as a competitive inhibitor but also affected its expression. More importantly, we found that inhibiting $\mathrm{HO}$ activity using the chemical inhibitor SnPP resulted in a loss of the vascular protective actions of rapamycin thus verifying the role of HO-1 in mediating rapamycin's antiproliferative effects in vascular disease. This study further demonstrates the cytoprotective effects of HO-1 in ameliorating pulmonary vascular disease. We found that the chemical inducer of HO, CoPP, resulted in a dramatic improvement of monocrotaline-induced pulmonary vascular disease.

Right-sided heart failure is a poor prognostic factor for survival in patients with pulmonary hypertension. ${ }^{1}$ Initially, RVH is an adaptive response to maintain right heart function against an elevated PVR, and HO-1 may play a role in this adaptive response. HO-1-deficient mice with hypoxia-induced pulmonary hypertension develop right-sided heart infarcts, a feature not observed in wild-type mice. ${ }^{19}$ Our studies did not show any differences in RVH with the addition of SnPP. Animals treated with rapamycin failed to increase right ventricular size in the face of an elevated $\mathrm{PaP}$, which may be detrimental for survival, although, no mortality was observed in any of the experimental groups at 28 days post monocrotaline exposure. Previously, rapamycin was found to attenuate pressure overload-induced cardiac hypertrophy. ${ }^{30}$ Similarly, we also found that rapamycin blocked the development of RVH in response to monocrotaline. However, unlike the pulmonary vasculature where SnPP inhibited rapamycin's effects, in cardiac muscle there was no effect on rapamycin's growth inhibition. This suggests that rapamycin antiproliferative actions are different on pulmonary vascular SMC and cardiac muscle cells.
Our previous study in HPASMC demonstrated an increase in HO-1 at only relatively higher doses of rapamycin. ${ }^{14}$ In addition, we found that the highly selective inhibitor, LY294004, of PI-3K, an upstream signaling molecule of the mTOR pathway, had no effect on HO-1 expression (data not shown). These data suggest that the induction of HO-1 by rapamycin may not involve mTOR pathway. This was further confirmed by the fact that rapamycindependent HO-1 induction was similar in mutant mTOR and wild-type Rh30 cells.

Since HO-1 expression is a sensitive marker for oxidant stress, ${ }^{25}$ and rapamycin altered the redox state of HPASMC, we tested the possibility that HO-1 induction by rapamycin was an oxidant-mediated event. The general antioxidant, NAC, blocked rapamycin-mediated increase in oxidants; however, NAC had only partial effect on rapamycin-dependent HO-1 induction, indicating a redox-independent mechanism. These findings are similar to HO-1 induction by growth factors such as transforming growth factor- $\beta$ in renal epithelial cells, wherein NAC is unable to block HO-1 induction. ${ }^{31}$

To verify that the findings observed using the chemical inhibitor SnPP is related to its inhibition of HO-1, we evaluated SMC derived from both wildtype and $\mathrm{HO}_{-1} 1^{-1-}$ mice. Since the vascular protective effects of rapamycin have been shown in both systemic and pulmonary vascular SMC, $\mathrm{SM}^{4,7,8,14}$ we isolated aortic SMC and evaluated potential mechanisms for the loss of rapamycin's antiproliferative properties. This study using vascular SMC derived from $\mathrm{HO}-1^{-1-}$ mice verifies the critical role of HO-1 in mediating the vascular antiproliferative actions of rapamycin. SMC deficient in HO-1 were not responsive to the antiproliferative or cell cycle inhibition actions of rapamycin.

One possibility for the ineffectiveness of rapamycin in HO-1-deficient cells is that these cells have an altered expression of cell cycle proteins. As discussed above, rapamycin is best known for inhibiting mTOR, ${ }^{32}$ and its inhibition of cell cycle progression was shown to be mediated by increasing the cell cycle inhibitory protein, p2 $7^{\mathrm{kip} 1},{ }^{26}$ thereby inhibiting cell cycle progression. In addition, HO-1 overexpression is known to inhibit cell growth and this is associated with an increase in the inhibitory protein $\mathrm{p} 21^{\mathrm{cip}} \cdot{ }^{33,34}$ We found that $\mathrm{p} 27^{\mathrm{kip} 1}, \mathrm{p} 21^{\mathrm{cip}}$, and cyclin D expression in response to growth factors and rapamycin were similar in wild-type and HO$1^{-/-}$mSMC. So unlike HO-1 overexpression, HO-1 deficiency effects are not mediated through p21 ${ }^{\text {cip }}$. The possibility that HO-1 and rapamycin interaction is not through rapamycin's inhibition of mTOR is further verified with the $\mathrm{p} 27^{\mathrm{kip} 1}$ results, since rapamycin effects on $\mathrm{p} 27^{\mathrm{kip} 1}$ are downstream to mTOR.

Recent studies have shown that products of HO-1 activity, carbon monoxide or biliverdin/bilirubin, inhibit SMC proliferation by activating p38 $\beta$ mitogenactivated protein kinase. $^{35,36}$ Downstream effects of both products result in an inhibition of cell 
cycle-dependent proteins with carbon monoxide altering caveolin expression and subsequently p2 ${ }^{\text {cip }}{ }^{35}$ while bilirubin effects may be mediated by inhibition of phosphorylation of the retinoblastoma tumor suppressor protein (Rb). ${ }^{36}$ Carbon monoxide and/or bilirubin may also mediate HO-1 effects in pulmonary hypertension, and future studies are planned to evaluate these products along with HO-1 actions on heme/iron metabolism.

Rapamycin treatment differentially express $\sim 10 \%$ of the genes in smooth muscle cells. ${ }^{37}$ These include cell cycle proteins such as the transcription factor E2F-1, and various Rb/E2F-1-regulated genes such as cyclin D3 and YY1 transcription factor. ${ }^{37}$ Similarly, HO-1 through the generation of its byproducts also inhibits the expression of several cell cycle proteins and inhibits hyperphosphorylation of $\mathrm{Rb}^{36}$ In addition, YY1 which regulates cell growth is also inhibited by bilirubin treatment. ${ }^{36}$ Furthermore, we found that rapamycin action on HO-1 expression is not mediated through mTOR, which requires rapamycin binding to FKBP12. Rapamycin potentially may bind to the another FKBP, such as FKBP25, which is known to alter DNA-binding activity of YY1. ${ }^{38}$ The similar actions of HO-1 and rapamycin on the Rb/E2F-1/YY1 pathway suggest that this pathway may be involved in rapamycin's interaction with $\mathrm{HO}-1$, and will be tested in the future.

Rapamycin has been shown to attenuate atherosclerosis in apoplipoprotein-deficient mice in a

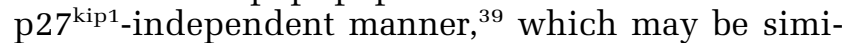
lar to our finding in which the lack of rapamycin antiproliferative effects in HO-1-deficient cells does not appear to be mediated through p27 $7^{\mathrm{kip} 1}$. In addition to its effects on cell cycle proteins, rapamycin inhibited the upregulation of monocyte chemotactic protein-1 (MCP-1) in these mice. ${ }^{39}$ Interestingly, HO-1 overexpression also protects apolipoprotein-deficient mice ${ }^{40}$ while a HO-1 deficiency exacerbates atherosclerosis. ${ }^{41} \mathrm{HO}-1$ also inhibits the upregulation of MCP- $1,{ }^{42}$ suggesting another potential interaction between rapamycin and $\mathrm{HO}-1$.

In summary, this study demonstrates that HO-1 plays an important role in mediating the vascular protective effects of rapamycin in both pulmonary and systemic vasculature. HO-1-deficient SMC are not sensitive to the antiproliferative effects of rapamycin in vitro and in vivo. The loss of HO-1 results in rapamycin resistance, although this does not appear to be mediated through it usual effects on mTOR nor alteration of cell cycle proteins (p21 ${ }^{\text {cip }}$, $\mathrm{p} 27^{\mathrm{kip} 1}$, or cyclin D1) involved in the actions of HO-1 and rapamycin.

\section{References}

1 Runo JR, Loyd JE. Primary pulmonary hypertension. Lancet 2003;361:1533-1544.
2 Rabinovitch M. Pulmonary hypertension: pathophysiology as a basis for clinical decision making. J Heart Lung Transplant 1999;11:1041-1053.

3 Shirotani M, Yui Y, Hattori R, et al. U-61,431F, a stable prostacyclin analogue, inhibits the proliferation of bovine vascular smooth muscle cells with little antiproliferative effect on endothelial cells. Prostaglandins 1991;41:97-110.

4 Marx SO, Jayaraman T, Go LO, et al. Rapamycin-FKBP inhibits cell cycle regulators of proliferation in vascular smooth muscle cells. Circ Res 1995;76:412-417.

5 Cao W, Mohacsi P, Shorthouse R, et al. Effects of rapamycin on growth factor-stimulated vascular smooth muscle cell DNA synthesis. Inhibition of basic fibroblast growth factor and platelet-derived growth factor action and antagonism of rapamycin by FK506. Transplantation 1995;59:390-395.

6 Vinals F, Chambard JC, Pouyssegur J. p70 S6 kinasemediated protein synthesis is a critical step for vascular endothelial cell proliferation. J Biol Chem 1999;274:26776-26782.

7 Burke SE, Lubbers NL, Chen YW, et al. Neointimal formation after balloon-induced vascular injury in Yucatan minipigs is reduced by oral rapamycin. J Cardiovasc Pharmacol 1999;33:829-835.

8 Suzuki T, Kopia G, Hayashi S, et al. Stent-based delivery of sirolimus reduces neointimal formation in a porcine coronary model. Circulation 2001;104: 1188-1193.

9 Colombo A, Moses JW, Morice MC, et al. Randomized study to evaluate sirolimus-eluting stents implanted at coronary bifurcation lesions. Circulation 2004;109: 1244-1249.

10 Nishimura T, Faul JL, Berry GJ, et al. 40-O-(2-hydroxyethyl)-rapamycin attenuates pulmonary arterial hypertension and neointimal formation in rats. Am J Resp Crit Care Med 2001;163:498-502.

11 Bruner LH, Bull RW, Roth RA. The effect of immunosuppressants and adoptive transfer in monocrotaline pyrrole pneumotoxicity. Toxicol Appl Pharmacol 1987;91:1-12.

12 Vaquero E, Molero X, Tian X, et al. Myofibroblast proliferation, fibrosis, and defective pancreatic repair induced by cyclosporin. Gut 1999;45:269-277.

13 Terada N, Lucas JJ, Szepesi A, et al. Rapamycin blocks cell cycle progression of activated $\mathrm{T}$ cells prior to events characteristic of the middle to late $G_{1}$ phase of the cycle. J Cell Physiol 1993;154:7-15.

14 Visner GA, Lu F, Zhou H, et al. Rapamycin induces heme oxygenase-1 in human pulmonary vascular cells: implications in the antiproliferative response to rapamycin. Circulation 2003;107:911-916.

15 Maines MD. Heme oxygenase: function, multiplicity, regulatory mechanisms, and clinical applications. FASEB J 1988;2:2257-2568.

16 Otterbein LE, Bach FH, Alam J, et al. Carbon monoxide has anti-inflammatory effects involving the mitogenactivating protein kinase pathway. Nat Med 2000;6: $422-428$

17 Liu X, Chapman GB, Wang $\mathrm{H}$, et al. Adenvirusmediated heme oxygenase-1 gene expression stimulates apoptosis in vascular smooth muscle cells. Circulation 2002;105:79-84.

18 Minamino T, Christou H, Hsieh CM, et al. Targeted expression of heme oxygenase- 1 prevents the pulmonary inflammatory and vascular responses to hypoxia. Proc Nat Acad Sci USA 2001;98:8798-8803. 
19 Yet SF, Perrella MA, Layne MD, et al. Hypoxia induces severe right ventricular dilatation and infarction in heme oxygenase-1 null mice. J Clin Invest 1999;103: R23-R29.

20 Ohar JA, Waller KS, Williams TJ, et al. Computerized morphometry of the pulmonary vasculature over a range of intravascular pressures. Anat Rec 1998;252:92-101.

21 Hosoi H, Dilling MB, Shikata T, et al. Rapamycin causes poorly reversible inhibition of mTOR and induces p53-independent apoptosis in human rhabdomyosarcoma cells. Cancer Res 1999;59:886-894.

22 Poss KD, Tonegawa S. Reduced stress defense in heme oxygenase 1-deficient cells. Proc Natl Acad Sci USA 1997;94:10925-10930.

23 Kapturczak MH, Wasserfall C, Brusko T, et al. Heme oxygenase-1 modulates early inflammatory responses: evidence from the heme oxygenase-1-deficient mouse. Am J Pathol 2004;165:1045-1053.

24 Hill-Kapturczak N, Voakes C, Garcia J, et al. A cis-acting region regulates oxidized lipid-mediated induction of the human heme oxygenase-1 gene in endothelial cells. Arterioscler Thromb Vasc Biol 2003;23:1416-1422.

25 Golden CL, Nick HS, Visner GA. Thrombin regulation of endothelin-1 gene in isolated human pulmonary endothelial cells. Am J Physiol 1998;18:L854-L863.

26 Castro C, Campistol JM, Sancho D, et al. Rapamycin attenuates atherosclerosis induced by dietary cholesterol in apolipoprotein-deficient mice through a p27 Kip1-independent pathway. Atherosclerosis 2004;172: 31-38.

27 Sikorski EM, Hock T, Hill-Kapturczak N, et al. The story so far: molecular regulation of the heme oxygenase-1 gene in renal injury. Am J Physiol 2004;286: F425-F441.

28 Tyrrell RM, Basu-Modak S. Transient enhancement of heme oxygenase 1 mRNA accumulation: a marker of oxidative stress to eukaryotic cells. Methods Enzymol 1994;234:224-235.

29 Christou H, Morita T, Hsieh CM, et al. Prevention of hypoxia-induced pulmonary hypertension by enhancement of endogenous heme oxygenase- 1 in the rat. Circ Res 2000;86:1224-1229.

30 Shioi T, McMullen JR, Rarnavski O, et al. Rapamycin attenuates load-induced cardiac hypertrophy in mice. Circulation 2003;107:1664-1667.

31 Hill-Kapturczak N, Truong L, Thamilselvan V, et al. Smad7-dependent regulation of heme oxygenase- 1 by transforming growth factor-beta in human renal epithelial cells. J Biol Chem 2000;275:40904-40909.

32 Seghal SN. Rapamune: Mechanism of action of immunosuppressive effect results from blockade of signal transduction and inhibition of cell cycle progression. Clin Biochem 1998;31:335-340.

33 Abraham NG, Scapagnini G, Kappas A. Human heme oxygenase: cell cycle-dependent expression and DNA microarray identification of multiple gene responses after transduction of endothelial cells. J Cell Biochem 2003;90:1098-1111.

34 Inguaggiato P, Gonzalez-Michaca L, Croatt AJ, et al. Cellular overexpression of heme oxygenase-1 upregulates p21 and confers resistance to apoptosis. Kidney Int 2001;60:2181-2191.

35 Kim HP, Wang X, Nakao A, et al. Caveolin-1 expression by means of p38beta mitogen-activated protein kinase mediates the antiproliferative effect of carbon monoxide. Proc Natl Acad Sci USA 2005;102:11319-11324.

36 Ollinger R, Bilban M, Erat A, et al. Bilirubin: a natural inhibitor of vascular smooth muscle cell proliferation. Circulation 2005;112:1030-1039.

37 Zohlnhofer D, Nuhrenberg TG, Neumann FJ, et al. Rapamycin effects transcriptional programs in smooth muscle cells controlling proliferative and inflammatory properties. Mol Pharmacol 2004;65:880-889.

38 Yang WM, Yao YL, Seto E. The FK506-binding protein 25 functionally associates with histone deacetylases and with transcription factor YY1. EMBO J 2001;20: 4814-4825.

39 Castro C, Campistol JM, Sancho D, et al. Rapamycin attenuates atherosclerosis induced by dietary cholesterol in apolipoprotein-deficient mice through a p27 Kip1-independent pathway. Atherosclerosis 2004;172: 31-38.

40 Juan SH, Lee TS, Tseng KW, et al. Adenovirusmediated heme oxygenase-1 gene transfer inhibits the development of atherosclerosis in apolipoprotein E-deficient mice. Circulation 2001;104:1519-1525.

41 Yet SF, Layne MD, Liu X, et al. Absence of heme oxygenase-1 exacerbates atherosclerotic lesion formation and vascular remodeling. FASEB J 2003;17: 1759-1761.

42 Nath KA, Vercellotti GM, Grande JP, et al. Heme protein-induced chronic renal inflammation: suppressive effect of induced heme oxygenase-1. Kidney Int 2001;59:106-117. 